\title{
A MPCC-NLP Approach for an Electric Power Market Problem
}

\author{
Helena Sofia Rodrigues ${ }^{1}$, Maria Teresa Torres Monteiroº ${ }^{2}$, António Ismael Freitas Vaz ${ }^{2}$ \\ ${ }^{1}$ School of Business Studies, Viana do Castelo Polytechnic Institute, Valença, Portugal; ${ }^{2}$ Department of Production and Systems, \\ University of Minho, Braga, Portugal \\ Email: sofiarodrigues@esce.ipvc.pt, \{tm, aivaz\}@dps.uminho.pt
}

Received November $21^{\text {st }}, 2009$; revised May $14^{\text {th }}, 2010$; accepted May $14^{\text {th }}, 2010$.

\begin{abstract}
The electric power market is changing-it has passed from a regulated market, where the government of each country had the control of prices, to a deregulated market economy. Each company competes in order to get more cli.e.nts and maximize its profits. This market is represented by a Stackelberg game with two firms, leader and follower, and the leader anticipates the reaction of the follower. The problem is formulated as a Mathematical Program with Complementarity Constraints (MPCC). It is shown that the constraint qualifications usually assumed to prove convergence of standard algorithms fail to hold for MPCC. To circumvent this, a reformulation for a nonlinear problem (NLP) is proposed. Numerical tests using the NEOS server platform are presented.
\end{abstract}

Keywords: Electric Power, Stackelberg Game, Nonlinear Programming, Complementarity Constrained Optimization

\section{Introduction}

The electric power market is in transition. Until recently, the market was regulated by the government of each country, and companies could only sell to a restrict set of consumers.

With the deregularization, electricity industry becomes a liberalized activity where planning and operation scheduling are independent activities which are not constrained by centralized procedures. On the other side, the generator firms take more risk as they become responsible for their decisions.

While in a regulated market the industry goal was to minimize the costs - once the price was fixed-now, it is also to maximize profit. A competition environment is created in order to benefit the consumers through price reduction, but ill effects can occur if the level of concentration in the market grows.

In order to study the interaction of all market participants and to have a better knowledge of the market conditions, firms and governments need suitable decisionsupport models. The deregularization process is under way in many countries. In 1998, the USA has begun their transition: California, Massachusetts and Rhode Island were the first states, but others will follow them over the next years. Nowadays, America's electric power industry is highly fragmented [1].

In Europe, the process has started in the decade of 80 in
England and Wales. In the last years, the market has been faced with fusions and merges between companies. The directives of European Union for an electric power liberality led up to increasing institutional and physical connections between markets from different countries. Some papers about studies in course, related with German, French and The Netherlands power markets-see [2-6] for more details-have emerged.

According to [7], there are reasons to consider electric power as a special commodity:

All power travels over the same set of power lines, independently of the firm that generated it; this difference is particularly marked when the networks contains loops and there are transmission capacity limits; also electricity has unique physical properties, namely Kirchhoff voltage and current laws.

As the electricity is difficult to store, and the quantity of power must be instantly adjusted to the demand, the companies that lead the market could easily manipulate the price, changing it to higher values, especially in peak consumption periods. The scientific community try to find models to predict how the prices will react to this new market structure.

The organization of this paper is as follows: Section 2 introduces the Stackelberg game and the related concepts and definitions of optimization. In Section 3, it is present the formulation of the electric power problem as well as its transformation from MPCC into a MPCC-NLP prob- 
lem and also the data specifying the network for the computational experiments. Finally, the numerical results obtained by a set of solvers are shown and the main conclusions are discussed in the last section.

\section{Stackelberg Game and Optimization}

To simulate the decision making process for defining offered prices in a deregulated environment, it was used the game theory, in particular the Stackelberg game. A parallelism between this economic theory and optimization is also addressed.

\subsection{Stackelberg Game}

In Stackelberg game there are two kinds of players: the leader and the followers. The leader firm has the power to manipulate the prices, production and expansion capacity in order to maximize its own profit and anticipates the reaction of the rest of the player firms. The leader uses the knowledge of the reactions in order to choose its own optimal strategy. The follower decisions are dependent on the leader strategy. The follower does not have the perception how its decisions influence the leader resolution.

Between followers their behaviour act like a noncooperative Nash game, where all players have the same information and no one can increase their own profit through unilateral decisions [8].

A Stackelberg game can be formulated as a bilevel programming problem and therefore we introduce the reader to it in the next subsection.

\subsection{Bilevel Optimization}

\section{Definition 1 Bilevel Optimization Problem}

A bilevel optimization problem is composed by a first-level problem:

$$
\begin{array}{cc}
\min _{x, y} & F_{1}(x, y) \\
\text { s.t. } & g(x, y) \leq 0
\end{array}
$$

Where $y$, for each value of $x$, is the solution of the second-level problem:

$$
\begin{array}{cc}
\min _{y} & F_{2}(x, y) \\
\text { s.t. } & h(x, y) \leq 0
\end{array}
$$

with $x \in I R^{n x}, y \in I R^{n y}, F_{1}, F_{2}: I R^{n x+n y} \rightarrow I R, g$ : $I R^{n x+n y} \rightarrow I R^{n u}, h: I R^{n x+n y} \rightarrow I R^{n l}$.

The variables $x[y]$ are called as first [second] level variable, $g(x, y)[h(x, y)]$ are the first [second] level constraints and $F_{1}(x, y)\left[F_{2}(x, y)\right]$ are the first [second] level objective function.

A typical bilevel problem is an optimization model whose constraints require that certain of its variables $(x)$ solve an optimization subproblem that dependents parametrically on the remaining variables $(y)$.

Regarding with careful attention the structure of the bilevel problem, it is possible to observe that the first/second level of the bilevel problem corresponds to the leader/followers players on the Stackelberg game.

A bilevel problem is convex if $F_{2}$ and $h$ are convex functions in $y$ for all values of $x$ that is to say if the second level problem is convex [9]. The problem studied in this paper is a convex bilevel problem. The great advantage of this property in bilevel optimization is that, under certain conditions, the second level problem can be replaced by their own Karush-Kuhn-Tucker (KKT) conditions, and the resulting problem is one level optimization problem with complementarity constraints.

\subsection{Mathematical Program with Complementarity Constraints}

\section{Definition 2 MPCC Problem}

Mathematical Program with Complementarity Constraint (MPCC) is defined as:

$$
\begin{array}{cc}
\min _{z} & F(z) \\
& c_{i}(z)=0, \quad i \in E \\
\text { s.t. } & c_{i}(z) \geq 0, \quad i \in I \\
& 0 \leq z_{1} \perp z_{2} \geq 0
\end{array}
$$

where $z=\left(z_{0}, z_{1}, z_{2}\right)$, with the control variable $z_{0} \in$ $I R^{n}$ and the state $z_{1}, z_{2} \in I R^{p} ; \mathrm{F}$ is the objective function, $c_{i}, i \in E \cup I$ are the set of equality and inequality constraints, respectively. The sets $E$ and $I$ are the finite sets of indices. The objective function $\mathrm{F}$ and the constraints $c_{i}, i \in E \cup I$ are assumed twice continuously differentiable. The constraints related to complementarity are defined with the operator $\perp$ and demand that the product of the two nonnegative quantities must be zero, i.e. $z_{1 i} z_{2 i}=0, i \in\{1, \ldots, p\}$.

The concept of complementarity distinguishes an MPCC from a standard nonlinear optimization problem and is a synonymous of equilibrium, reason why this type of problem is so popular in optimization (see $[8,10,11]$ for some applications in the last years).

In engineering, the MPCC problems are being used for contact and structural mechanic problems, namely in robotic [3,12,13], obstacle problems [14], elastohydrodinamic lubrification $[15,16]$, process engineering models [17] and traffic network equilibrium $[18,19]$.

Applications in economics include the general equilibrium and game theory from which Nash and Stackelberg game are instances [20-22].

A new field of applications is in ecological problems: the questions related with reduction of greenhouse gas emission rights, coalition formation and international trade in order to negotiate the emission rights between develop and developing countries can be also formulated 
as a MPCC problem [23,24].

The MPCC problem is nonsmooth mostly due to the complementarity constraints. The optimal conditions are complex and very difficult to verify. Besides, the feasible set of MPCC is ill-posed since the constraint qualifications - namely, Mangasarian Fromovitz (MFCQ) and Linear Independent (LICQ)-which are commonly assumed to prove convergence of standard nonlinear programming do not hold at any feasible point of the complementarity constraints $[25,26]$. This implies mostly that the multiplier set is unbounded, the active constraint normal are linearly dependent and the linearizations of the MPCC can become inconsistent arbitrarily close to a solution.

The violation of constraint qualifications has led to a number of specific algorithms for MPCCs, such as branch-and-bound [27], implicit nonsmooth approaches [28], piecewise SQP methods [8] and perturbation and penalization approaches [29]. But the use of specific solvers for MPCC is not a real solution at this time, since these algorithms still need rather strong assumptions to ensure convergence.

The search of new techniques and algorithms in order to solve real problems with large dimension is still an area with intense research. Recently, some authors suggested solving MPCC problem by an interesting way: reformulated it into an equivalent NLP problem. This formulation allows taking advantage of certain NLP algorithms features in order to obtain rapid local convergence. Besides, it works like a challenge for the NLP solver, because it allows testing its reliability and robustness, whereas the MPCC problem has specific irregularities.

A MPCC defined in (3) can be reformulated as an equivalent NLP problem:

$$
\begin{gathered}
\text { Definition } 3 \text { NLP formulation of the MPCC problem } \\
\min _{z} \\
F(z) \\
c_{i}(z)=0, i \in E \\
c_{i}(z) \geq 0, \quad i \in I \\
\text { s.t. } \\
z_{1} \geq 0 \\
z_{2} \geq 0 \\
z_{1}^{\mathrm{T}} z_{2} \leq 0
\end{gathered}
$$

Recall that the complementarity constraint was replaced by a nonlinear inequality, relaxing the problem. The transformation from a MPCC problem into a NLP problem allows using standard NLP solvers taking to advantage of the convergence properties of these solvers.

One can easily show that the reformulated problem has the same properties that the previous one, including constraint qualifications and second-order conditions, which means that the violation of MFCQ is still a reality. However, in the last few years, some studies show that strong stationarity is equivalent to the KKT conditions of the
MPCC-NLP problem [30,31]. This fact has advantages because strong stationarity is a useful and practical computation characterization, once it is relatively easy to find a stationary point in a NLP solver, under reasonable assumptions.

\section{The Electric Power Market Problem}

The problem described in this paper is based on the model proposed in [32]. It is a competitive power market, formulated as an oligopolistic equilibrium model.

There are a number of generator firms, each owing a given number of units. These make an hourly bid to an Independent System Operator (ISO). The ISO, taking in consideration the network, solves a social welfare maximization problem, announces a dispatch for each bidder and possibly distinct prices at each node. It decides how much power to buy from generators and how much power to distribute to consumers and what prices to charge. All these decisions are made with the optimal power flow (OPF) in mind.

In spite of the fact that the ISO expects the bid to be a reflex of the true costs, the reality is different: the units, generally, increase their own bid, without the knowledge of the outside world, as the Figure 1 shows. This strategy has as main goal the increase of the units' profit.

The leader generator first decides and takes as input all the perceptions and information that it could have about the market (including predictable bids of the other firms, demand and supply functions) and it maximizes its profit inside a set of spatial price equilibrium constraints and Kirchhoff's voltage and current laws. The followers firms make their own decisions taking into account the leader decision.

\subsection{Formulation}

In [32] the electric power market was formulated as a bilevel problem. In the first level-the leader level-the parameter related with the bid curve corresponds to the first level variable. In the second level-the follower level there is a simulation of the conjectures of the market promoted by ISO and can be described as a commodity spatial price equilibrium problem. The model tries to find the optimal bid for each company.

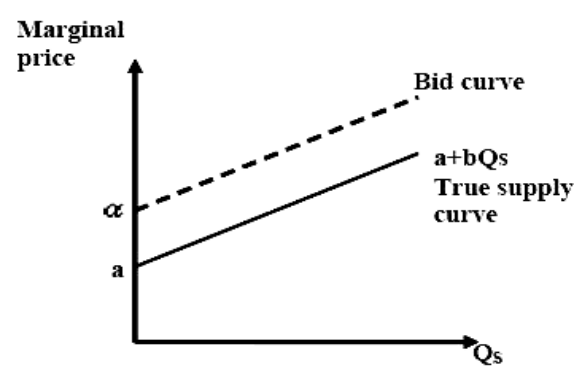

Figure 1. Marginal cost and bid curves. 
Next we introduce the notation used in the mathematical formulation:

Indices:

$i$

$i j$

node in the network

arc from node $i$ to node $j$

m

number of Kirchhoff voltage loops in the network

\section{Sets:}

$N \quad$ set of all nodes

A set of all arcs

$S_{f} \quad$ set of generator nodes under control of leader firm $f$

$P \quad$ set of all generators nodes

D set of all demand nodes

$L \quad$ set of Kirchhoff' voltage loops $m$

$L_{m} \quad$ set of ordered arcs (clockwise) associated with loop $m$

Recall that, a node can be, simultaneously a generator and a consumer, so $P$ and $D$ are not necessarily disjoint and their union could be a proper subset of $N$. The uniqueness of the net flow on each arc is ensured by the Kirchhoff's laws in the linearized DC models and, consequently, the number of (independent) loops is $\# A-\# N+$ 1 (where $\# X$ is the set $X$ cardinality).

Parameters:

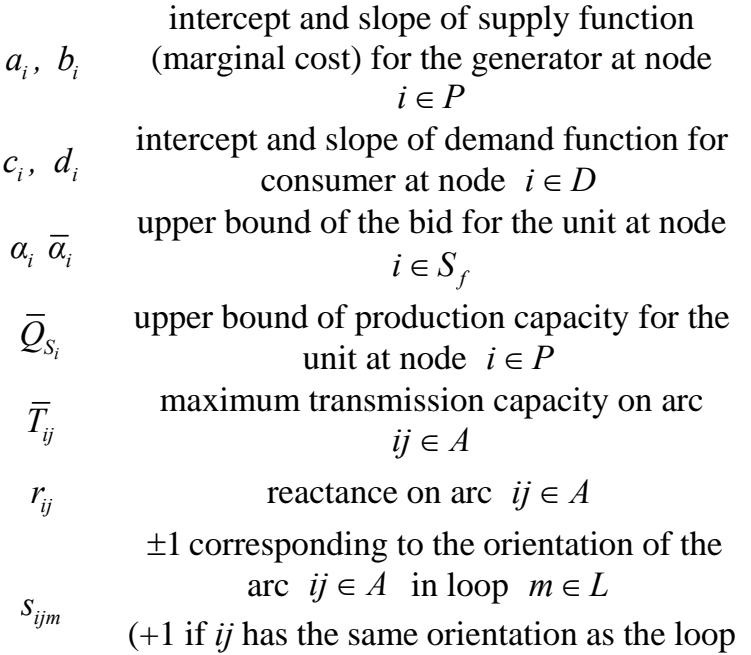
$m)$

First-Level decision variable

$\alpha_{i} \quad$ bid for the unit at node $i \in P$

In this model, it is assumed that the generate firms can only manipulate $\alpha$ (the intercept in the bid function) and not the slope $b$, due to market and optimization assumptions.

Let $\alpha_{i}$ be fixed for the competitive firms (i.e. $\alpha_{i}$ fixed $\forall i \in P \mid S_{f}$ ) and variables for the leader firms (i.e. $\alpha_{i}$ variable $\forall i \in S_{f}$ ).

Primal variables in the second-level: vector defined by quantity of power generated by the unit at node $i$

$$
\begin{gathered}
Q_{S_{i}} \quad\left(Q_{S_{i}}=a_{i}+b_{i} Q_{S_{i}} \text { if } i \in P \text { and } Q_{S_{i}}=0\right. \text { if } \\
i \notin P)
\end{gathered}
$$

quantity of power demanded at node $i$

$Q_{D_{i}} \quad\left(Q_{D_{i}}=c_{i}-d_{i} Q_{D_{i}}\right.$ if $i \in D$ and $Q_{D_{i}}=0$ if $i \notin D)$

$T_{i j} \quad$ matrix defined by MW transmitted from node $i$ to node $j$

Dual variables in the second-level

$\lambda_{i} \quad$ marginal cost at node $i$

$\mu_{i} \quad$ marginal value of generation capacity for unit

$\mu_{i} \quad$ at node $i$

$\theta_{i j} \quad$ marginal value of transmission capacity on arc ij

shadow price for Kirchhoff voltage law for loop $m$

Next, it is defined the second-level convex quadratic problem. The objective function is related with the maximization of social welfare:

$$
\max \sum_{i \in D}\left(c_{i} Q_{D_{i}}-\frac{1}{2} d_{i} Q_{D_{i}}^{2}\right)-\sum_{i \in P}\left(\alpha_{i} Q_{S_{i}}+\frac{1}{2} b_{i} Q_{S_{i}}^{2}\right)
$$

This function reports a solution where the firms maximize their profits and the consumers maximize the utility of the product.

The following constraints report to a spatial price equilibrium plus a constraint due to Kirchhoff voltage law.

- Nonnegative demand variables:

$Q_{D_{i}} \geq 0, \quad i \in D$

- Lower and upper bounds for transmission variables: $0 \leq T_{i j} \leq \bar{T}_{i j}, \quad i j \in A$

- Minimum and maximum capacity of production:

$0 \leq Q_{S_{i}} \leq \bar{Q}_{S_{i}}, \quad i \in P$

- Conservation constraints:

$Q_{D_{i}}-Q_{S_{i}}+\sum_{j: i j \in A} T_{i j}-\sum_{j: i j \in A} \bar{T}_{i j}=0$

- Kirchhoff voltage law:

$\sum_{i j \in L m} s_{i j m} r_{i j} T_{i j}=0$

If in Equation (8), by economic reasons, the minimum production level could not be zero, it is possible to change the lower bound and still use the same model.

The description of the first level of the electric power is complete by taking into account that for the follower firms the bids are already fixed. The determination of the dominant company profit consists in finding a bid vector $\alpha^{f} \equiv\left(\alpha_{i}: i \in S_{f}\right)$, a vector of supplies $Q_{S}$, a vector of demands $Q_{D}$ and a vector of transmission capacities $T$, by solving the following maximization problem.

$$
\text { Maximize } \quad \pi(\lambda, Q) \equiv\left(\lambda Q-a Q-\frac{b}{2} Q\right)
$$




$$
\text { s.t. } \quad 0 \leq \alpha \leq \bar{\alpha}, \quad \forall i \in S
$$

where $Q_{S}, Q_{D}$ and $T$ for each value of $\alpha \equiv(\alpha: i \in P)$, are the solution of the second-level problem (5-10).

It is provided in [33] that, for each vector $\alpha$, there exists a unique globally optimal solution of the quadratic problem above.

But, solving a bilevel problem is not an easy task. So, the approach is to replace the ISO's lower-level optimization problem by its stationary conditions that results in a system of equilibrium constraints. To write the above information into a vector-matrix notation, it is necessary to introduce two additional matrices.

Let $\Delta$ be the matrix which give us the information about the pair (node, arc) in the electric network:

$$
\Delta_{i l}=\left\{\begin{array}{ccc}
1, & \text { if } l=i j \in A & \text { for some } j \in N \\
-1, & \text { if } l=i j \in A & \text { for some } j \in N \\
0, & \text { other values } &
\end{array}\right.
$$

Let $R$ be the matrix (arc, cycle) related with the reactance coefficients:

$$
R_{i j m}=\left\{\begin{array}{cl}
s_{i j m} r_{i j}, & \text { if } i j \in L_{m} \\
0, & \text { otherwise }
\end{array}\right.
$$

So, the Karush-Kuhn-Tucker (KKT) optimality conditions of the lower problem are:

$$
\begin{array}{ccc}
0 \leq \bar{Q}_{S}-Q_{S} & \perp & \mu \geq 0 \\
0 \leq Q_{S} & \perp & -\lambda+\mu+\alpha+\operatorname{diag}(b) Q_{S} \geq 0 \\
0 \leq Q_{D} & \perp & \lambda-c+\alpha+\operatorname{diag}(d) Q_{\mathrm{D}} \geq 0
\end{array}
$$

$$
\begin{array}{lcc}
0 \leq \theta & \perp & \bar{T}-T \geq 0 \\
0 \leq T & \perp & \Delta^{T} \lambda+\theta+R \gamma \geq 0 \\
\lambda \text { free } & & Q_{D}-Q_{S}+\Delta T=0 \\
\gamma \text { free } & & R^{T} T=0
\end{array}
$$

where $\mu, \theta, \lambda$ and $\gamma$ are the dual variables. The notation $\operatorname{diag}(w)$ represents the diagonal matrix whose diagonal entries are the components of the vector $w$.

Then, the second-level problem (5-10) can be replaced by the KKT conditions (14) and the MPCC problem is obtained by joining (11) and (14).

For computational reasons the objective function needs to be reformulated, since it is neither convex nor concave due to the term $\lambda_{i} Q_{S_{i}}$. The equivalent objective function for solve the maximization of the leader firm profit is:

$$
\begin{gathered}
\pi_{\mathrm{f}}\left(\lambda, \mathrm{Q}_{\mathrm{S}}\right) \equiv \sum_{i \in D}\left(c_{i} Q_{D_{i}}-d_{i} Q_{D_{i}}^{2}\right)-\sum_{i \in S_{f}}\left(a_{i} Q_{S_{i}}-\frac{b_{i}}{2} Q_{S_{i}}^{2}\right) \\
-\sum_{i j \in A} \theta_{i j} \bar{T}_{i j}-\sum_{i \in P \mid S_{f}}\left(\mu_{i} \bar{Q}_{S_{i}}+a_{i} Q_{S_{i}}-b_{i} Q_{S_{i}}^{2}\right)
\end{gathered}
$$

\subsection{Data}

The electric power network includes a circuit with 30 nodes, which 6 are nodes with generators- 3 for the leader firm A and the 3 remaining for the follower firm B-and 21 are demand nodes. Connecting the nodes there are 41 arcs and 12 loops. Figure 2 shows a scheme that provides all the necessary information.

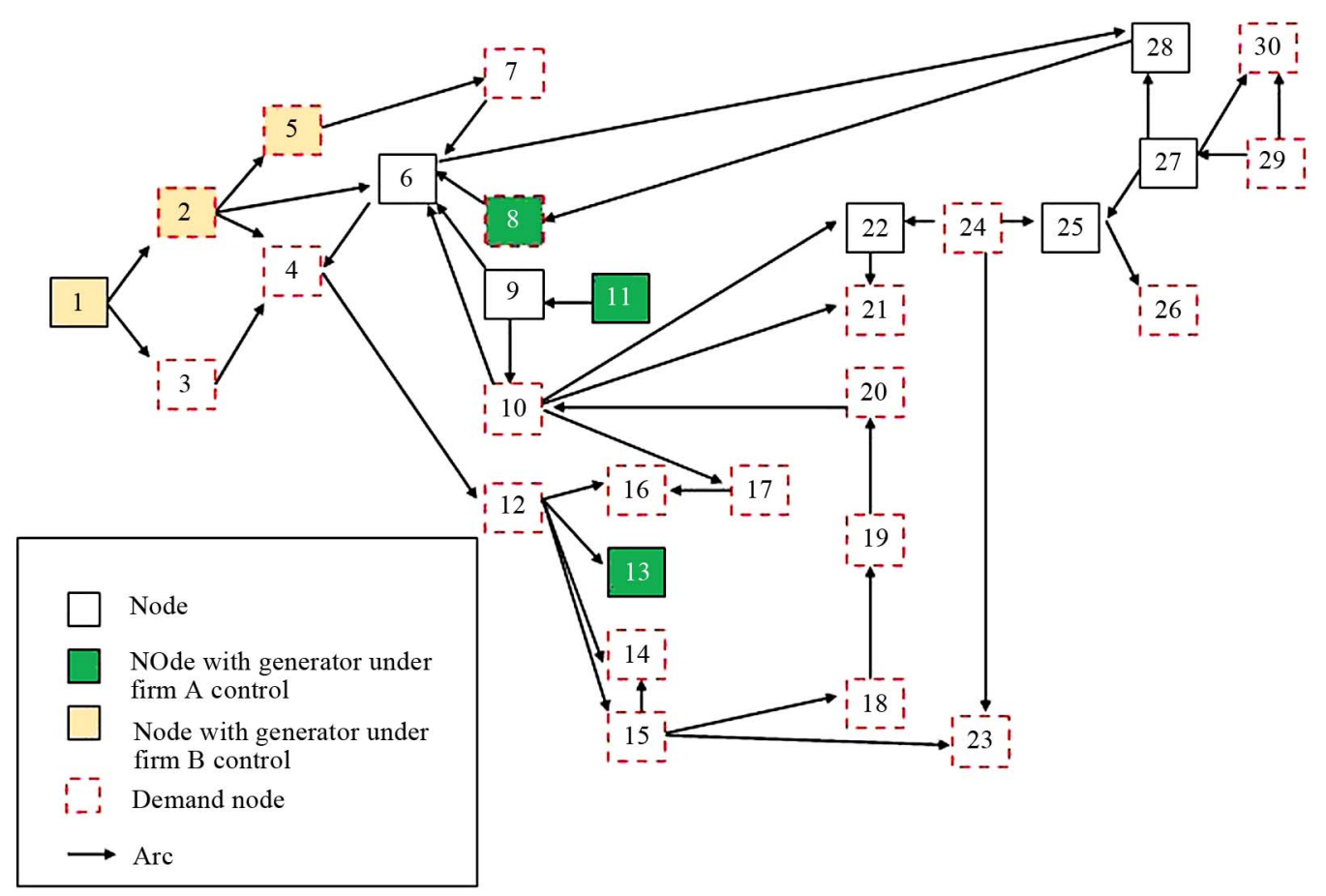

Figure 1. Electric Network 
The data related with production, demand, transmission values are based on [7]. The generator cost function, reactance and upper bounds for supply and transmission flows values are also given. As a safety measure of the network the upper bounds values for the transmission capacity are $60 \%$ of the values assumed in [8].

To solve the dominant firm A problem it is assumed that the bids for the units of the company $\mathrm{B}$ are equals to their marginal costs, which means $\alpha=a$.

The demand curve for each costumer node is determined by $P_{i}=40-d_{i} Q_{D_{i}}$ where $d_{i}$ is chosen so that $P_{i}$ $=\$ 30 / M W h$ when $Q_{D_{i}}$ equals the value assumed in [34].

The code of this problem is in AMPL language and can be found in the MacMPEC [35] with the name monteiro.mod. It is a problem with 136 variables, 201 constraints where 62 of them are complementarity constraints.

To solve the problem, the MPCC-NLP approach was used, meaning that all complementarity constraints were reformulated as nonlinear constraints according the definition (4).

\section{Computational Results and Conclusions}

To solve the electric power problem it were used three nonlinear solvers that have distinct characteristics.

Lancelot [36] is a standard Fortran 77 package for large scale nonlinear optimization, developed by Conn, Gould and Toint. The software uses an augmented Lagrangian approach and combines a trust region approach adapted to handle the bound constraints.

Loqo [37] was developed by Vanderbei and is a software for solving smooth constrained optimization problems. It is based on an infeasible primal-dual interior point method applied to a sequence of quadratic approximations. It uses line search to induce global convergence and the Hessian is exact.

The Snopt, developed by Gill, Murray and Saunders, is a software package for solving large-scale linear and nonlinear programs. The functions used should be smooth but not necessary convex and it is especially effective for problems whose functions and gradients are expensive to evaluate.

The NEOS Server [38] platform was used to interface with the selected solvers. NEOS (Network Enabled Optimization System) is an optimization service that is available through the Internet. It is a large set of software packages considered as the state of the art in optimization.

The numerical results obtained by the used NLP solvers are presented in Table $\mathbf{1}$ where the objective functions together with the first level variables are shown.

Curiously, although it has been reached an identical value for all solvers for the objective function, the same didn't happen for the bid variable, which take us to believe for the existence of the several local maximum points.

For the second-level variables the values are also different, as the Tables $\mathbf{2}$ and $\mathbf{3}$ expose.

There are some demand nodes that practically do not receive electric power. This may be explained for two reasons: economical ones because it is possible that the transportation of the energy for these places are too ex pensive and by the existence of large demander nodes close to the generator units that absorbed all the power

Table 1. Objective function and bid results

\begin{tabular}{ccc}
\hline Solver & $\begin{array}{c}\text { Profit function } \\
(\pi)\end{array}$ & $\operatorname{Bid}\left(\alpha^{\mathrm{f}}\right)$ \\
\hline LANCELOT & 37.53 & $(35.83,40,29.80)$ \\
LOQO & 37.53 & $(35.83,36.09,20)$ \\
SNOPT & 37.53 & $(35.83,39.99,0)$ \\
\hline
\end{tabular}

Table 2. Demanded power

\begin{tabular}{|c|c|c|c|c|c|c|c|}
\hline Node & Lancelot & Loqo & Snopt & Node & Lancelot & Loqo & Snopt \\
\hline 2 & 44.98 & 44.98 & 44.98 & 17 & 0 & $-1.37 e-14$ & 0 \\
\hline 3 & 2.55 & 2.55 & 2.55 & 18 & 0 & $-4.54 \mathrm{e}-15$ & $-2.28 \mathrm{e}-26$ \\
\hline 4 & 6.87 & 6.87 & 6.87 & 19 & 0 & $-4.55 e-15$ & $2.94 \mathrm{e}-13$ \\
\hline 5 & 41.04 & 41.04 & 41.04 & 20 & 0 & $-4.71 e-15$ & $-5.98 e-26$ \\
\hline 7 & 0 & $-1.43 e-14$ & 0 & 21 & 0 & 0 & 0 \\
\hline 8 & 10.01 & 10.01 & 10.01 & 23 & 0 & -6.42 & $2.96 \mathrm{e}-13$ \\
\hline 12 & 0 & $-1.41 \mathrm{e}-14$ & 1.09 & 26 & 0 & 0 & 0 \\
\hline 14 & 0 & $1.35 \mathrm{e}-14$ & 0 & 29 & 0 & 0 & 0 \\
\hline 15 & 0 & $-5.71 e-15$ & $-2.96 e-13$ & 30 & 0 & 0 & 0 \\
\hline 16 & $1.32 \mathrm{e}-05$ & $-4.32 \mathrm{e}-15$ & $5.11 \mathrm{e}-13$ & & & & \\
\hline
\end{tabular}

Table 3. Generated power

\begin{tabular}{ccccccc}
\hline Node & 1 & 2 & 5 & 8 & 11 \\
\hline Lancelot & 44.30 & 10.09 & 41.04 & 10.01 & $1.29 \mathrm{e}-5$ & 13 \\
Loqo & 44.31 & 10.09 & 41.04 & 1.01 & 0 \\
Snopt & 44.31 & 10.09 & 41.04 & 10.01 & $-2-16 \mathrm{e}-13$ & 0 \\
\hline
\end{tabular}


produced.

It has been shown that MPCC-NLP approach should be considered to solve real problems.

As future work it is proposed the study of this problem developed as a nash model, where both firms compete at the same level and with the same market information.

\section{EFERENCES}

[1] M. Willrich, "Electricity Transmission Policy for America: Enabling a Smart Grid, End to End," The Electricity Journal, Vol. 22, No. 10, 2009, pp. 77-82.

[2] "Third Benchmarking Report on the Implementation of the Internal Electricity and Gas Markets,” Tech Republic 1, Commission Draft Staff Paper, March 2004.

[3] B. F. Hobbs and F. A. M. Rijkers, "Strategic Generation with Conjectured Transmission Price Responses in a Mixed Transmission Pricing System," IEEE Transmissions on Power Systems, Vol. 19, No. 2, 2004, pp. 707-879.

[4] Y. Smeers, "How Well Can Measure Market Power in Restructured Electricity Systems,” Tech Republic, University Catholique De Louvain, November 2004.

[5] S. A. Gabriel and F. U. Leuthold, "Solving DiscretelyConstrained MPEC Problems with Applications in Electric Power Markets," Energy Economics, Vol. 32, No, 1, 2010, pp. 3-14.

[6] M. Crappe, “Electric Power Systems,” Wiley-ISTE, 2009.

[7] C. B. Metzler, "Complementarity Models of Competitive Oligopolistic Electric Power Generation Markets,” Master’s Thesis, The Johns Hopkins University, 2000.

[8] Z.-Q. Luo, J.-S. Pang and D. Ralph, "Mathematical Programs with Equilibrium Constraints," Cambridge University Press, Cambridge, 1996.

[9] L. N. Vicente and P. H. Calamai, "Bilevel and Multilevel Programming: a Bibliography Review,” Journal of Global Optimization, Vol. 5, No. 3, 1994, pp. 291-306.

[10] S. Dempe, “Annotated Bibliography on Bilevel Programming and Mathematical Programs with Equilibrium Constraints,” Optimization, Vol. 52, No. 3, 2003, pp. 333-359.

[11] M. C. Ferris and J. S. Pang, "Engineering and Economic applications of Complementarity Problems," SIAM Review, Vol. 39, No. 4, 1997, pp. 669-713.

[12] M. F. Ferris and F. Tin-Loi, "Limit Analysis of Frictional Block Assemblies as a Mathematical Program with Complementarity Constraints," International Journal of Mechanical Sciences, Vol. 43, No. 1, 2001, pp. 209-224.

[13] J. S. Pang, “Complementarity Problems,” In: R. Horst and P. Pardalos, Eds., Handbook in Global Optimization, K. A. Publishers, Boston, 1994.

[14] J. F. Rodrigues, "Obstacle Problems in Mathematics PhySics,” Elsevier Publishing Company, Amsterdam, 1987.

[15] M. C. Ferris and F. Tin-Loi, "On the Solution of a Minimum Weight Elastoplastic Problem Involving Displacement and Complementarity Constraints," Computer Methods in Applied Mechanics and Engineering, Vol. 174, No. 1-2, 1999, pp. 107-120.
[16] K. P. Oh and P. K. Goenka, "The Elastohydrodynamic Solution of Journal Bearings under Dynamic Loading," Transactions of American Society of Mechanical Engineers, Vol. 107, No. 3, 1985, pp. 389-395.

[17] A. U. Raghunathan and L. T. Biegler, "Mathematical Programs with Equilibrium Constraints (Mpecs) in Process Engineering," Tech Republic, CMU Chemical Engineering, November 2002.

[18] O. Drissi-Kaitouni and J. Lundgren, "Bilivel Origin-Destination Matrix Estimation Using a Descent Approach,” Tech Republic, Linköping Institute of Technology, Depart-ment of Mathematics, Sweden, 1992.

[19] M. Stohr, "Nonsmooth Trust Region Methods and their Applications to Mathematical Programs with Equilibrium Constraints," Shaker Verlag, Aachen, 2000.

[20] C. Ballard, D. Fullerton, J. B. Shoven and J. Whalley, "A General Equilibrium Modelfor Tax Policy Evaluation,” National Bureau of Economic Research Monograph, Chicago, 1985.

[21] H. Ehtamo and T. Raivio, "On Aapplied Nonlinear and Bilevel Programming for Pursuit-Evasion Games,” Journal of Optimization Theory and Applications, Vol. 108, No. 1, 2001, pp. 65-96.

[22] J. V. Outrata, "On Necessary Optimal Conditions for Stackelberg Problems," Journal of Optimization Theory and Applications, Vol. 76, No. 2, 1993, pp. 305-320.

[23] J. C. Carbone, C. Helm and T. F. Rutherford, "Coalition formation and International Trade in Greenhouse Gas Emission Rights," MIMEO, the Sixth Annual Conference on Global Economic Analysis, Scheveningen, The Hague, The Netherlands, June 12-14 2003.

[24] G. Hibino, M. Kainuma and Y. Matsuoka, "Two-Level Mathematical Programming for Analysing Subsidy Options to Reduce Greenhouse-Gas Emissions,” Tech Republic, Laxenburg, 1996.

[25] M. Kocvara and J. V. Outrata, "Optimization Problems with Equilibrium Constraints and their Numerical Solution,” Mathematical Programs, Series B, Vol. 101, No. 1, 2004, pp. 119-149.

[26] H. S. Rodrigues and M. T. Monteiro, "Solving Mathematical Program with Complementarity Constraints (MPCC) with Nonlinear Solvers," Recent Advances in Optimization, Lectures Notes in Economics and Mathematical Systems, Springer, Berlin, Part 4, 2006, pp. 415424

[27] J. F. Bard, “Convex Two-Level Optimization,” Mathematical Programming, Vol. 40, No. 1, 1988, pp. 15-27.

[28] J. Outrata, M. Kocvara and J. Zowe, "Nonsmooth Approach to Optimization Problems with Equilibrium Constraints,” Kluwer Academic Publishers, Dordrecht, 1998.

[29] M. C. Ferris, S. P. Dirkse and A. Meeraus, "Mathematical Programs with Equilibrium Constraints: Automatic Reformulation and Solution via Constrained Optimization,” Tech Republic, Oxford University Computing Laboratory, July 2002.

[30] H. Scheel and S. Scholtes, "Mathematical Programs with 
Complementarity Constraints: Stationarity, Optimality and Sensitivity," Mathematics of Operations Research, Vol. 25, No. 1, 2000, pp. 1-22.

[31] S. Scholtes, "Convergence Properties of a Regularization Scheme for Mathematical Programs with Complementarity Constraints," SIAM Journal Optmization, Vol. 11, No. 4, 2001, pp. 918-936.

[32] B. F. Hobbs, C. B. Metzler and J.-S. Pang, "Strategic Gaming Analysis for Electric Power Systems: An MPEC Approach," IEEE Transactions on Power Systems, Vol. 15, No. 2, 2000, pp. 638-645.

[33] V. Krishna and V. C. Ramesh, "Intelligent agents in NegotIations in Market Games, Part 2: Application,” IEEE Transactions on Power Systems, Vol. 13, No. 3, 1998, pp. 1109-1114.
[34] O. Alsa and B. Scott, "Optimal Load Flow with SteadyState Security," IEEE Transactions on Power Systems, Vol. 93, No. 3, 1973, pp. 745-751.

[35] S. Leyffer, Macmpec, 2010. http://www.mcs.nl.gov/ leyffer/macmpec

[36] Lancelot, 2010. http://www.numerical.rl.ac.uk/lancelot/blurb. html

[37] H. Benson, A. Sen, D. Shano and R. Vanderbei, "InteriorPoint Algorithms Penalty Methods and Equilibrium Problems,” Tech Republic, Operations Research and Financial Engineering Princeton University, October 2003.

[38] NEOS, 2010. http://www-neos.mcs.ang.gov/neos 